\title{
Upgrade of the proximity focusing RICH at JLab
}

\author{
E. Cisbani ${ }^{\text {a,* }}$, S. Colilli ${ }^{\text {a }}$, F. Cusanno ${ }^{\text {a }}$, R. Fratoni ${ }^{\text {a }}$, S. Frullani ${ }^{\text {a }}$, F. Garibaldi ${ }^{\text {a }}$, F. Giuliani ${ }^{\text {a }}$, M. Gricia ${ }^{\text {a }}$, \\ M. Lucentini ${ }^{a}$, F. Santavenere ${ }^{a}$, G.M. Urciuoli ${ }^{a}$, M. Iodice ${ }^{b}$, A. Argentieri ${ }^{c}$, G. De Cataldo ${ }^{c}$, R. De Leo $^{c}$, \\ L. Lagamba ${ }^{c}$, S. Marrone ${ }^{c}$, E. Nappi ${ }^{c}$, A. Camsonne ${ }^{d}$, B. Kross ${ }^{d}$, R. Michaels $^{d}$, B. Reitz ${ }^{\text {d }}$, J. Segal ${ }^{d}$, \\ B. Wojtsekhowski ${ }^{\mathrm{d}}$, C. Zorn ${ }^{\mathrm{d}}$, E. Monno ${ }^{\mathrm{e}}, \mathrm{H}$. Breuer ${ }^{\mathrm{f}}$ \\ a Istituto Superiore di Sanità and INFN Roma, I-00161 Rome, Italy \\ b INFN Roma Tre, I-00146 Rome, Italy \\ ${ }^{\mathrm{C}}$ University of Bari and INFN Bari, I-70126 Bari, Italy \\ ${ }^{\mathrm{d}}$ Thomas Jefferson National Accelerator Facility, Newport News, VA 23606, USA \\ e ENEA, Centro Ricerche Faenza, Faenza I-48018, Italy \\ ${ }^{\mathrm{f}}$ University of Maryland, College Park, MD 20742, USA
}

\section{A R T I C L E I N F O}

Available online 20 July 2008

Keywords:

Gas-filled counters

Cherenkov detector

Neutron spin structure

\begin{abstract}
A B S T R A C T
The Hall A RICH at Jefferson Lab is undergoing an upgrade to adapt to the higher momentum kinematics of the neutron spin structure Transversity experiments planned to run in 2008.

The JLab RICH is a proximity focusing detector using liquid $\mathrm{C}_{6} \mathrm{~F}_{14}$ as Cherenkov radiator, a thin layer of CSI as photon converter, evaporated on segmented pad panels of a proportional chamber. The original RICH had a superior hadron identification up to $2 \mathrm{GeV} / c$ with pion/kaon rejection at the level of 1:1000 at $\sim 90 \%$ intrinsic efficiency. The upgrade will extend this performance above $2.4 \mathrm{GeV} / \mathrm{c}$ by means of a larger photon detector (a multiwire-multipad proportional chamber) and a longer proximity gap which will improve the photon detection geometrical efficiency and the angular resolution, respectively.
\end{abstract}

(c) 2008 Elsevier B.V. All rights reserved.

\section{Original JLab RICH}

The experimental equipment of Hall A at Jefferson Lab includes a proximity focusing RICH detector [1] which has been originally designed to clearly identify pions, kaons and protons up to $2 \mathrm{GeV} / c$.

The JLab RICH is conceptually identical to the ALICE HMPID RICH [2]. As shown in Fig. 1, the Cherenkov light is generated in the liquid $\mathrm{C}_{6} \mathrm{~F}_{14}$ perfluoroalkane (1.28 refractive index) radiator contained in a fragile vessel made of Neoceram ${ }^{\circledR 1}$ on the entrance and lateral sides and $5 \mathrm{~mm}$ thick quartz slabs as the exit window. After the quartz, the photons propagate through a $\mathrm{CH}_{4}$-filled proximity gap of $10 \mathrm{~cm}$ length, and finally strike a $300 \mathrm{~nm}$ thin CsI layer on top of three pad panels. The CsI acts as photon-toelectron converter with a quantum efficiency better than $20 \%$ around the wavelength of $\lambda=160 \mathrm{~nm}$ [3]. The electrons migrate to the positive anode wire plane (at $2 \mathrm{~mm}$ from the pads), while the corresponding ion avalanche is slowly collected by one or more of the closest of the $11520\left(8 \times 8.4 \mathrm{~mm}^{2}\right)$ pads. The readout electronics connected to the pads is based on the track-and-hold multiplexed Gassiplex chip [4].

\footnotetext{
* Corresponding author.

E-mail address: evaristo.cisbani@iss.infn.it (E. Cisbani).

${ }^{1}$ NEOCERAM $^{\circledR}$ is a trademark of Nippon electric glass.
}

These components are mechanically assembled in a sandwich of six aluminum frames (Fig. 2). The liquid perfluoroalkane is continuously purified (oxygen and water molecules are the main components that degrade the transmittance) by a dedicated circulation system.

The CsI layer is obtained by the evaporation of CsI powder in a dedicated high vacuum chamber (few $10^{-7}$ mbar); the quality of the evaporation is controlled by an online quantum efficiency measurement device integrated in the evaporation chamber [5].

This configuration has operated successfully in the hypernuclear [6] and Pentaquark experiments in Hall A: $2 \mathrm{GeV} / \mathrm{c}$ hadrons have been identified with a $\pi: \mathrm{K}$ rejection better than $1: 1000$ (at $\sim 90 \%$ efficiency).

\section{RICH upgrade}

Two new experimental proposals concerning the transverse spin structure of the neutron have been approved by the JLab 29th Program Advisory Committee in January 2006. The new (Transversity) experiments [7] will measure the $2.4( \pm 5 \%) \mathrm{GeV} / \mathrm{c}$ $\pi^{ \pm}$and $\mathrm{K}^{ \pm}$single spin asymmetries of the semi-inclusive deep inelastic scattering of electrons on transversely polarized neutrons $\left({ }^{3} \mathrm{He}\right.$ target). Taking into account the larger pion production with respect to kaons $\left(N_{\pi} / N_{\mathrm{K}} \sim 10^{2}\right)$ and assuming 
that the pion-kaon asymmetries are of the same order, a $\pi: \mathrm{K}$ rejection of $\sim 10^{-3}$ is required to get a kaon asymmetry systematic uncertainty smaller or comparable to its statistical error.

Such a requirement has triggered the need to upgrade the RICH, according to the following guidelines:

- achieve the required performance: $\pi: \mathrm{K}$ rejection better than $1: 1000$ at $2.4( \pm 5 \%) \mathrm{GeV} / c$ with efficiency above $90 \%$;

- minimize the costs: maximize the use of existing parts;

- minimize the time: possibly use consolidate technology;

- preserve original performance.

Different options have been considered; two of them have been analyzed in more details:

(i) Use radiator with lower refractive index $\left(\mathrm{C}_{5} \mathrm{~F}_{12}\right.$ perfluoroalkane with $n=1.24$ ): lower $n$ means smaller angles (reduced

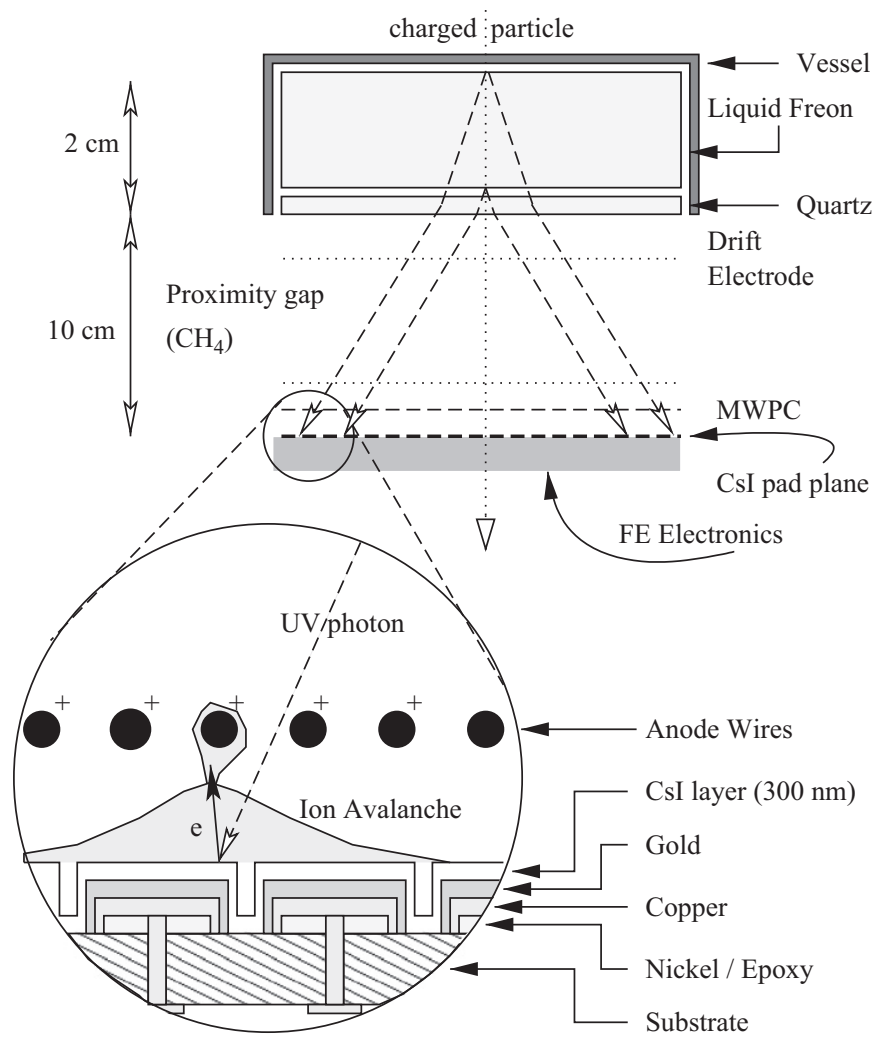

Fig. 1. Pictorial view of the JLab RICH: Cherenkov emission, propagation, photon conversion and charge collection.

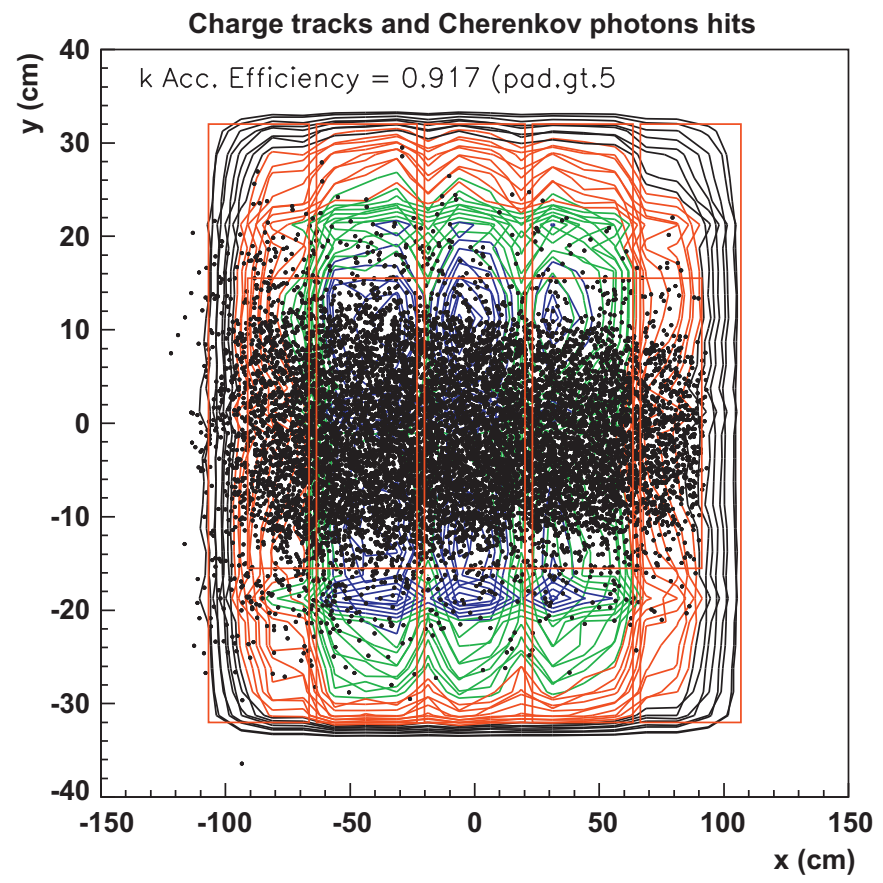

yg VS. $x g$

Fig. 3. Real particle track hits (black points) and simulated Cherenkov photons (contour lines); the inner box represents the radiator; the five vertical boxes correspond to the pad panels (not to scale).

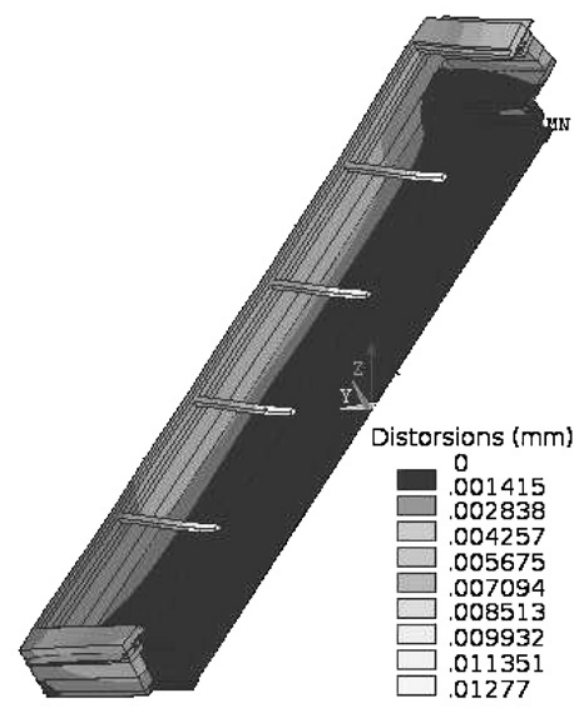

Fig. 4. Finite element analysis mechanical distortion.

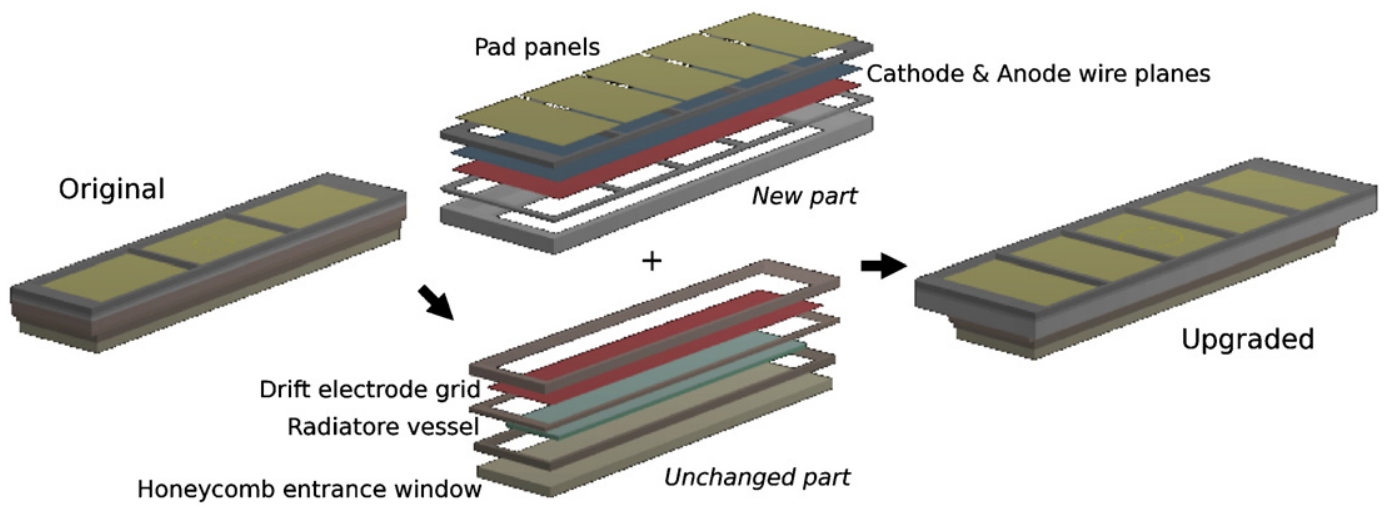

Fig. 2. 3D CAD views of the original (left) and the upgraded detector (right) with an exploded view (middle) where the unchanged and new parts are visible. 


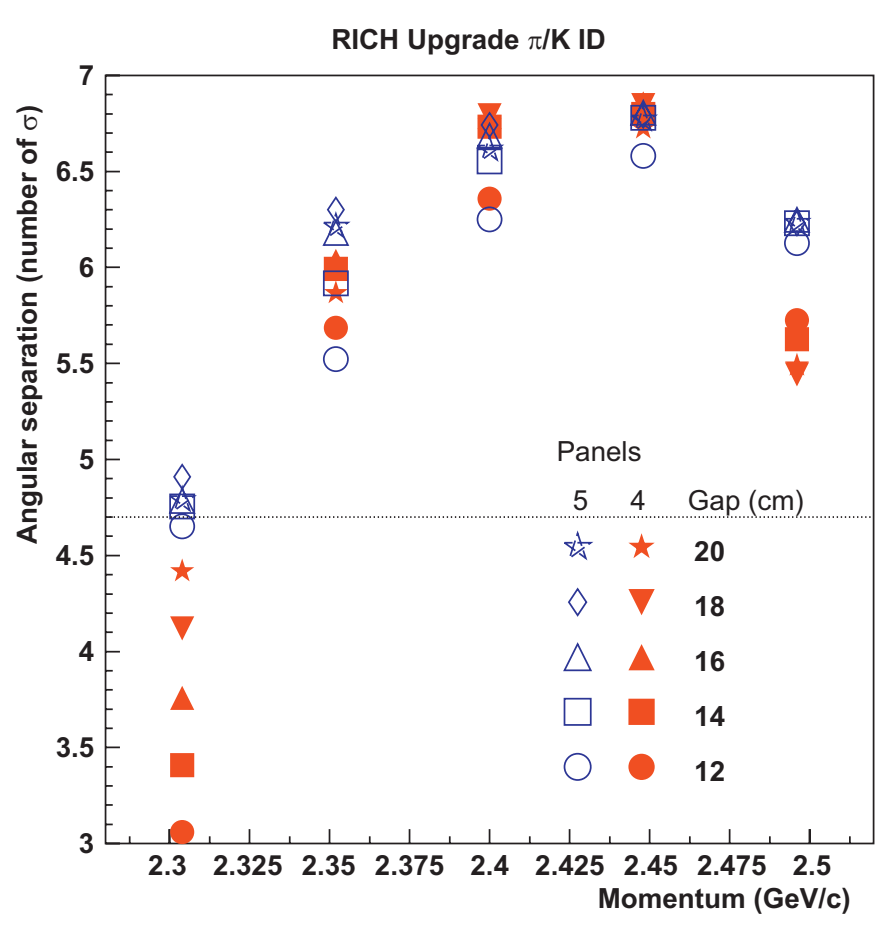

Fig. 5. Expected ID performance in terms of the distance between the angular distributions of the $\pi-\mathrm{K}$ Cherenkov photons. A separation of $4.7 \sigma$ (horizontal line) corresponds to $1: 1000 \pi: \mathrm{K}$ rejection.

number of photons but improved photon detector acceptance) and larger $\pi-\mathrm{K}$ Cherenkov angle difference. ${ }^{2}$

(ii) Extend the photon detection surface: as a design choice of the original RICH (to limit the number of electronics channels), part of the Cherenkov photons fall outside the detection plane. Therefore, a larger detector improves the photon collection, and at the same time permits to increase the proximity gap and consequently the angular resolution. ${ }^{3}$

While option (i) is expected to provide a slightly better performance, the $\mathrm{C}_{5} \mathrm{~F}_{12}$ has two relevant drawbacks:

- The pure quality is extremely expensive: there are cheap isomeric mixtures of $\mathrm{C}_{5} \mathrm{~F}_{12}$ whose transparency in the critical range of $160-220 \mathrm{~nm}$ is not guaranteed. ${ }^{4}$

- Its boiling point at standard pressure is close to $30^{\circ} \mathrm{C}$; it therefore requires a new circulation and cooling system and possibly a new thermally insulated vessel.

On the other hand, option (ii) does not present significant obstacles, does not require extensive R\&D and reuses a large part of the existing detector. Combining pros and cons of the two approaches and the upgrade constraints, the latter one has been chosen.

\subsection{Adopted solution in detail}

The original $\mathrm{RICH}$ detection planes is made by three rectangular pad panels, $640 \times 403 \mathrm{~mm}^{2}$ each, joined along their shorter side and therefore forming a surface of $1940 \times 403 \mathrm{~mm}^{2}(20 \mathrm{~mm}$ between panels) as shown in the left part of Fig. 2 .

In the upgraded version, the panels are joined along their longer side and two additional panels are added (see right picture in Fig. 2), obtaining a surface of $2050 \times 640 \mathrm{~mm}^{2}, 1.65$ times larger than the original. The new configuration has required the manufacturing of three new frames out of six as pictorially presented in the middle picture of Fig. 2.

The larger photon detection plane permits a longer proximity gap (from 100 to $175 \mathrm{~mm}$ ) and therefore a smaller overall angle reconstruction error. Charged tracks (from real events) and simulated photon hits distributions on the pad plane are presented in Fig. 3; efficiency larger than 90\% (photons detected on five or more pads, for each track) is expected.

The mechanical stress of the larger upgraded detector has been simulated by a detailed finite element model, tuned to the original RICH deformation data. The most significant result is shown in Fig. 4: the maximum distortion $(<0.02 \mathrm{~mm}$ ) expected on the thin roads between the pad panels is within the acceptable tolerance. ${ }^{5}$

The wires of the anode and cathode planes (at 2 and $4 \mathrm{~mm}$ from the pad plane) are anchored on Macor ${ }^{\circledR 6}{ }^{6}$ strips that are glued on the thin bars between the pad panels; each $2050 \mathrm{~mm}$ long wire acts as five shorter $(\sim 400 \mathrm{~mm})$ segments with fixed ends.

The expected performance has been estimated by a full GEANT3 simulation tuned on the original RICH results. Separation between pion and kaon angles, in the required momentum range, is presented in Fig. 5, for different gap lengths; two pad panel configurations have been simulated: the upgrade solution with five panels and a reduced 4 panel version. The latter is basically identical to the former in the central momentum region but degrades significantly at the lower and upper borders due to the shorter length of the detector (and therefore larger collection inefficiency). Detector alignment will improve the identification at the lowest momenta.

\section{Conclusions}

The upgrade activity is in progress (December 2007): the three new aluminum frames-which represent the most work-are almost complete; wire stretching, assembling and testing will immediately follow. The upgraded detector will operate late July 2008 when the Transversity experiments are scheduled for data taking.

\section{References}

[1] M. Iodice, et al., Nucl. Instr. and Meth. A 553 (2005) 231.

[2] Alice Collaboration, Technical Design Report of the High Momentum Particle Identification Detector CERN/LHCC98-19, 1998.;

L. Molnar, Nucl. Instr. and Meth. A 595 (2008) 27.

[3] Cusanno, et al., Nucl. Instr. and Meth. A 502 (2003) 251.

[4] J.C. Santiard, K. Marent, The Gassiplex0.7-2 integrated front-end analog processor for the HMPID and the dimuon spectrometer of ALICE, CERN/ ALICE-PUB 2001-49, 2001.

[5] E. Cisbani, et al., RICH detector at Jefferson Lab, design, performance and physics results, in: 9th Conference on Astroparticle, Particle and Space Physics, Detectors and Medical Physics Applications, Como, Italy, World Scientific, Singapore, 2005, pp. 36-41.

[6] M. Iodice, et al., Phys. Rev. Lett. 99 (2007) 052501/1-5.

[7] H. Gao, et al., Few-Body Syst. 41 (2007) 43.

\footnotetext{
${ }^{2}\left(\cos \theta_{\pi}-\cos \theta_{\mathrm{K}}\right) \sim\left(m_{\pi}^{2}-m_{\mathrm{K}}^{2}\right) /(2 n p)$, where $\theta$ is the Cherenkov angle, $m$ the mass, $p$ the particle momentum, $n$ the refractive index.

${ }^{3}$ Emission $\Delta \theta_{e} \sim w_{f} / w_{g}$ and sampling $\Delta \theta_{e} \sim L_{\text {pad }} /\left(w_{f}+w_{g}\right)$ angular errors are improved by longer $w_{\mathrm{g}}$ gap; here $L_{\mathrm{pad}}$ is the linear pad size, $w_{f}$ the radiator thickness.

${ }_{4}^{4}$ Transmittance of a $20 \%$ isomeric mixture of $C_{5} F_{12}$ sample has been measured to be about $\frac{1}{3}$ that of $\mathrm{C}_{6} \mathrm{~F}_{14}$.
}

\footnotetext{
5 The pad panel is made by two PCBs glued on a rigid aluminum frame, which improves the rigidity of the whole detector.

${ }^{6}$ Macor $^{\circledR}$ is a registered trademark of Cornig Incorporated.
} 